\title{
The bone marrow niche components are adversely affected in sepsis
}

\author{
Fan Yin ${ }^{1}$, Han Qian ${ }^{1}$, Caiwen Duan ${ }^{2^{*}}$ and Botao Ning ${ }^{1^{*}}$
}

\begin{abstract}
Multiple organ dysfunction is an important cause of death in patients with sepsis. Currently, few studies have focused on the impact of sepsis on bone marrow (BM), especially on the cell components of BM niche. In this study, we performed mouse sepsis models by intraperitoneal injection of LPS and cecal ligation and puncture (CLP). The changes of niche major components in the mouse BM among vascular structures, mesenchymal stem cells and Treg cells were observed and analyzed. The results showed that pathological changes in BM was earlier and more prominent than in other organs, and various cell components of the BM niche changed significantly, of which vascular endothelial cells increased transiently with vascular remodeling and the regulatory $T$ cells decreased over a long period of time. These results indicated that the components of the BM niche underwent series of adaptive changes in sepsis.

Keywords: Bone marrow niche, Sepsis, Lipopolysaccharide (LPS), Mouse model
\end{abstract}

\section{Introduction}

Sepsis is life-threatening organ dysfunction due to a dysregulated host response to infection, and is the leading cause of death in intensive care unit (ICU) [1, 2]. Septic shock frequently cause multi-organ dysfunction [3]. Among these affected organs, it is not clear which was affected initially and most significantly. Clinically, we tend to focus on the conditions of infection, sepsisinduced cardiomyopathy, disseminated intravascular coagulation, and the injury to respiratory system, liver and kidney, while ignored alterations of bone marrow (BM) during sepsis, especially the changes in BM niche.

Previous studies have suggested that key organs such as kidney and gut are vulnerable during sepsis $[4,5]$, interactions between organs during sepsis play a pivotal role in sepsis pathogenesis [3]. Notably, BM plays an equally important part in sepsis [6], which contains hematopoietic

\footnotetext{
* Correspondence: duancaiwen@163.com; ningbotao@126.com

${ }^{2}$ Department of Translational Institution, Shanghai Children's Medical Center, Shanghai Jiaotong University School of Medicine, 1678 Dongfang Road, Shanghai, China

'Department of Pediatric Intensive Care Unit, Shanghai Children's Medical Center, Shanghai Jiaotong University School of Medicine, 1678 Dongfang Road, Shanghai, China
}

progenitor cells and supporting niche cells. Sepsismediated BM suppression often leads to myeloid cell differentiation disorders, and myeloid cell dysfunction is associated with acquired immunodeficiency in sepsis [7]. Niche components of BM do not participate in hematopoiesis, but support hematopoiesis and maintain niche homeostasis. About sepsis, there has been so far much research focused on the regulation of the BM models by lipopolysaccharide (LPS) intraperitoneal injection and cecal ligation and puncture (CLP) to map the

\section{Results}

BM is earlier and more prominent in sepsis-mediated

To illustrate multiple organ damage in LPS treated mice, we observed the pathological changes of the main organs including BM, lung, kidney, liver, intestine and heart. In 
a

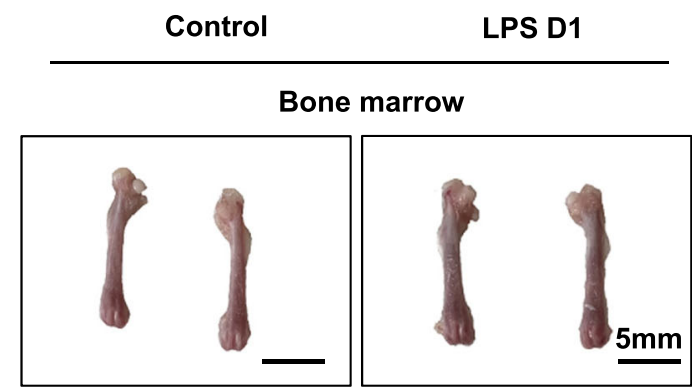

liver
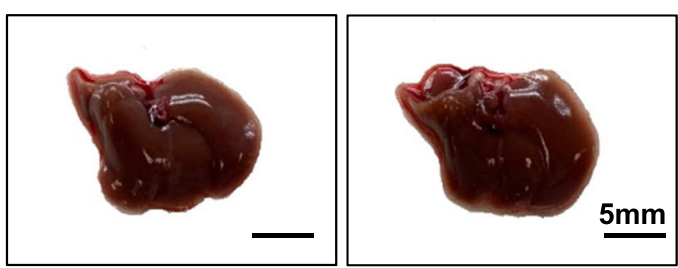

b
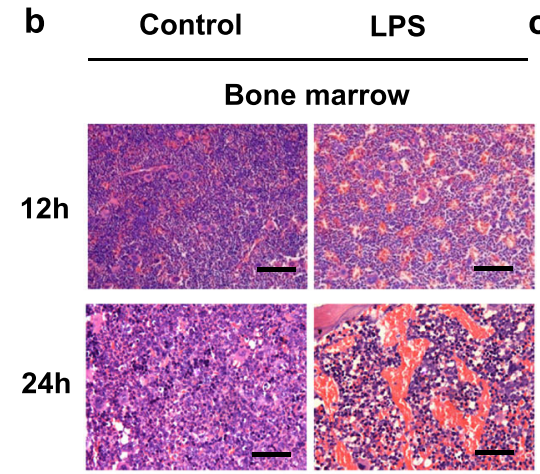

$72 \mathrm{~h}$

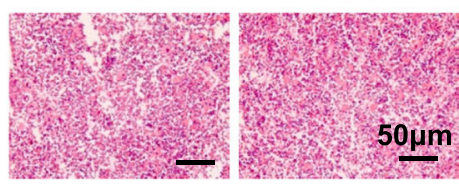

e

Liver
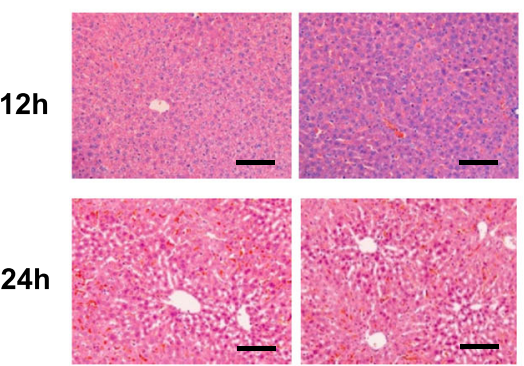

${ }^{72 n}$
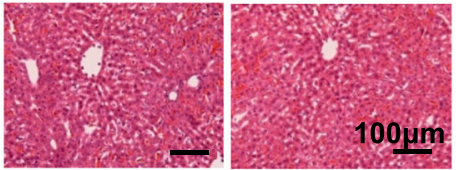

C Control
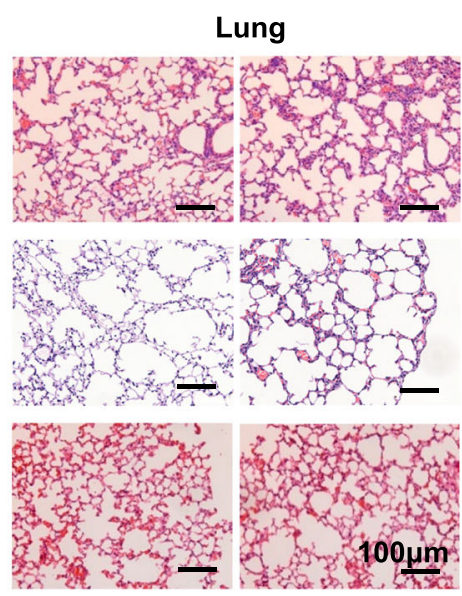

f

Intestine
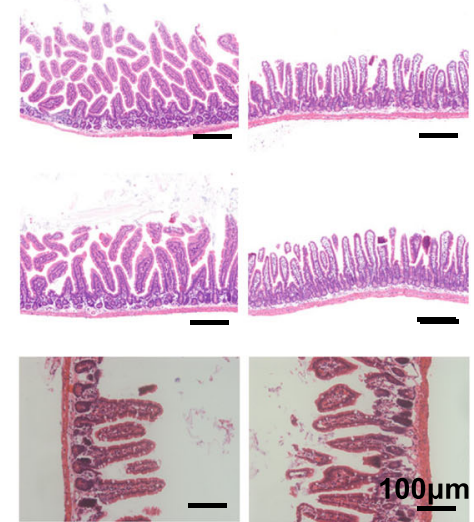

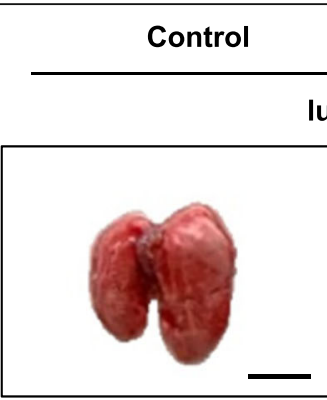

lung

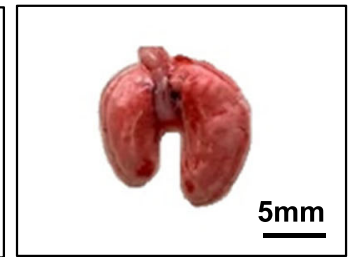

kidney

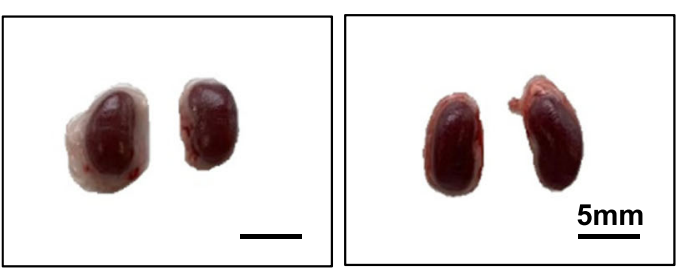

d Control

LPS

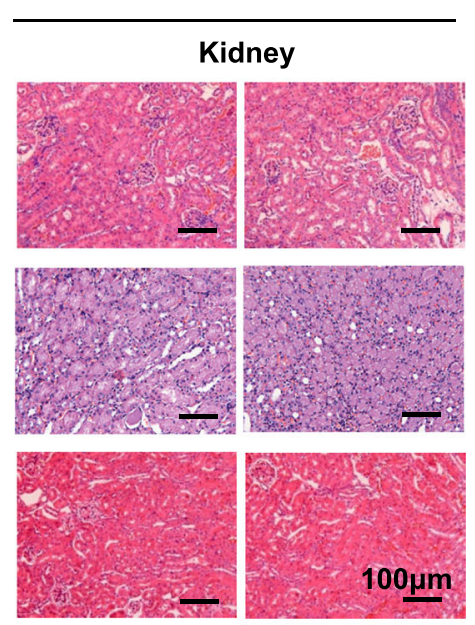

g

Heart

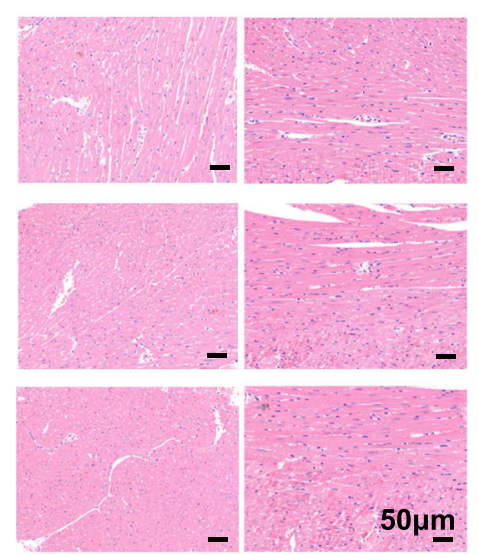

Fig. 1 (See legend on next page.) 
(See figure on previous page.)

Fig. 1 BM undergoes significant histological changes after LPS challenge. a Macroscopic images of BM, lung, liver and kidney from 8-week-old control (day 1 after PBS treatment) and LPS D1 (day 1 after LPS treatment) mice. $\mathbf{b}, \mathbf{c}, \mathbf{d}, \mathbf{e}, \mathbf{f}, \mathbf{g}$ Histological analysis by H\&E staining in the BM $(B M)(\mathbf{b})$, lung $(\mathbf{c})$, kidney $(\mathbf{d})$, liver $(\mathbf{e})$, intestine $(\mathbf{f})$ and heart $(\mathbf{g})$ of mice sepsis model prepared by intraperitoneal injection of LPS at $12 \mathrm{~h}, 24 \mathrm{~h}$ and $72 \mathrm{~h}$ after treatment. All representative pictures are verified by independent experiments $(n=3)$, both control and LPS-treated mice have biological replicates $(n>5)$

LPS treated mice, except for BM congestion, no obvious macroscopic changes were seen in these organs (Fig. 1a). We further studied the pathological changes of major organs in LPS treated mice from the histological level. LPS induced pathological changes in BM mainly occurred in vasculature. Compared with the PBS treated mice, LPS treated mice show obvious hyperemia at $12 \mathrm{~h}$ and BM vascular congestion peaked at $24 \mathrm{~h}$ and began to subside after $72 \mathrm{~h}$ (Fig. 1b). While the lung injury induced by intraperitoneal injection of LPS was relatively mild, and histopathological alterations mainly included pulmonary edema, pulmonary interstitial exudation, neutrophil infiltration (Fig. 1c). Next, we studied the histopathological alterations of the kidney, liver, intestine and heart of mice after LPS challenge and found that there were no obvious pathological changes in these organs (Fig. 1d-g). Taken together, BM was more susceptible to inflammation and exhibited histopathological alterations, which were different from other organs in early sepsis. Concomitantly, the histopathological changes of the main organs in mice after CLP were similar with those after LPS stimulation (Fig. 2a-d).

\section{Sepsis induces transient BM vasculature remodeling and BM EC proliferation}

BM vasculature changed drastically in previous histological analysis. To further monitor the structure of the $\mathrm{BM}$ vasculature, we specifically labeled the BM vessels by immunostaining. After $24 \mathrm{~h}$, compared with PBStreated mice, BM vasculature increased significantly in diaphysis regions of LPS and CLP treated mice as intuitively showed in anti-Endomucin staining of frozen BM sections (Fig. 3a). To quantify the LPS and CLP mediated $\mathrm{BM}$ vessel remodeling, we evaluated the $\mathrm{BM}$ vessel fluorescent area labeled by endomucin and the lumen area of the BM vessel. We found an increased fluorescence area and enlarged vasculature emerged $24 \mathrm{~h}$ following related treatment (Fig. 3b, c), a finding that correlated well with an increased branch in BM vasculature patterns of LPS treated mice (Fig. 3d, e). These support the obvious structural remodeling of BM vessels within a short time after LPS and CLP stimulation.

ECs are important cell types involved in vascular remodeling [10]. FACS analysis was used to determine the status of BM vascular ECs after LPS injection. We observed a rise in proliferating $\mathrm{Ki}^{+} 7^{+} \mathrm{BM}$ ECs $24 \mathrm{~h}$ after LPS injection (Fig. 3f, g), indicating that cells in S-phase increased. Consistent with this, there was a significant increase in frequency and absolute number of BM CD $45^{-}$Ter $119^{-} \mathrm{CD} 31^{+}$ECs of LPS treated mice in comparison to mice treated with PBS at $24 \mathrm{~h}$ (Fig. 3h, i). When mice were given sufficient time to recover after treatment, upregulation of BM ECs returned to homeostatic levels after $96 \mathrm{~h}$ (Fig. 3j). This indicated that the response of ECs to LPS treatment is transient. We further found even low dose $(1 \mathrm{mg} / \mathrm{kg})$ of LPS exposure could still activate BM ECs (Fig. 3k). This indicates that LPS stimulation activates ECs in a dose-independent manner and that BM ECs were activated even in response to low doses of treatment. Taken together, proliferation of BM ECs is one of the main causes of LPSmediated BM vascular remodeling.

\section{Sepsis did not significantly affect the frequency and location of BM MSCs}

To illustrate the effects of sepsis on BM perivascular stromal cells enriched in MSCs (MSC) activity, we used mice expressing GFP under the direction of nestin promoter (Nestin-GFP) [11]. Compared with mice treated with PBS, the femur vertical sections in mice treated with LPS revealed that the intensity and density of GFP fluorescence in both the epiphysis and diaphysis regions showed no obvious changes (Fig. 4a), which was consistent with the femur cross section slides in LPS treated mice (Fig. 4b). To quantify the LPS mediated MSCs changes, we evaluated the GFP fluorescent area and MSC spots counts of the BM cross section slides, and we founded there was no significant increase in both GFP fluorescent area and MSC spots counts after LPS treatment (Fig. 4c, d). Meanwhile, we also analyzed the spatial relationship between MSCs and vessels in BM and founded that the spatial distribution of MSCs was not affected by LPS stimulation, which mainly distributed around BM vessels (Fig. 4e). We next analyzed the frequency of MSCs using FACS and observed no significant increase in $\mathrm{CD}^{-} 5^{-}$Ter $119^{-} \mathrm{CD}^{-} 1^{-} \mathrm{GFP}^{+}$MSCs $24 \mathrm{~h}$ after LPS treatment (Fig. 4f, g), which is consistent with the visualization of BM frozen sections. Taken together, BM MSCs did not respond to LPS stimulation at the cellular and tissue level. In addition, the MSCs still mainly distributed around the vessels, close to the BM ECs. 


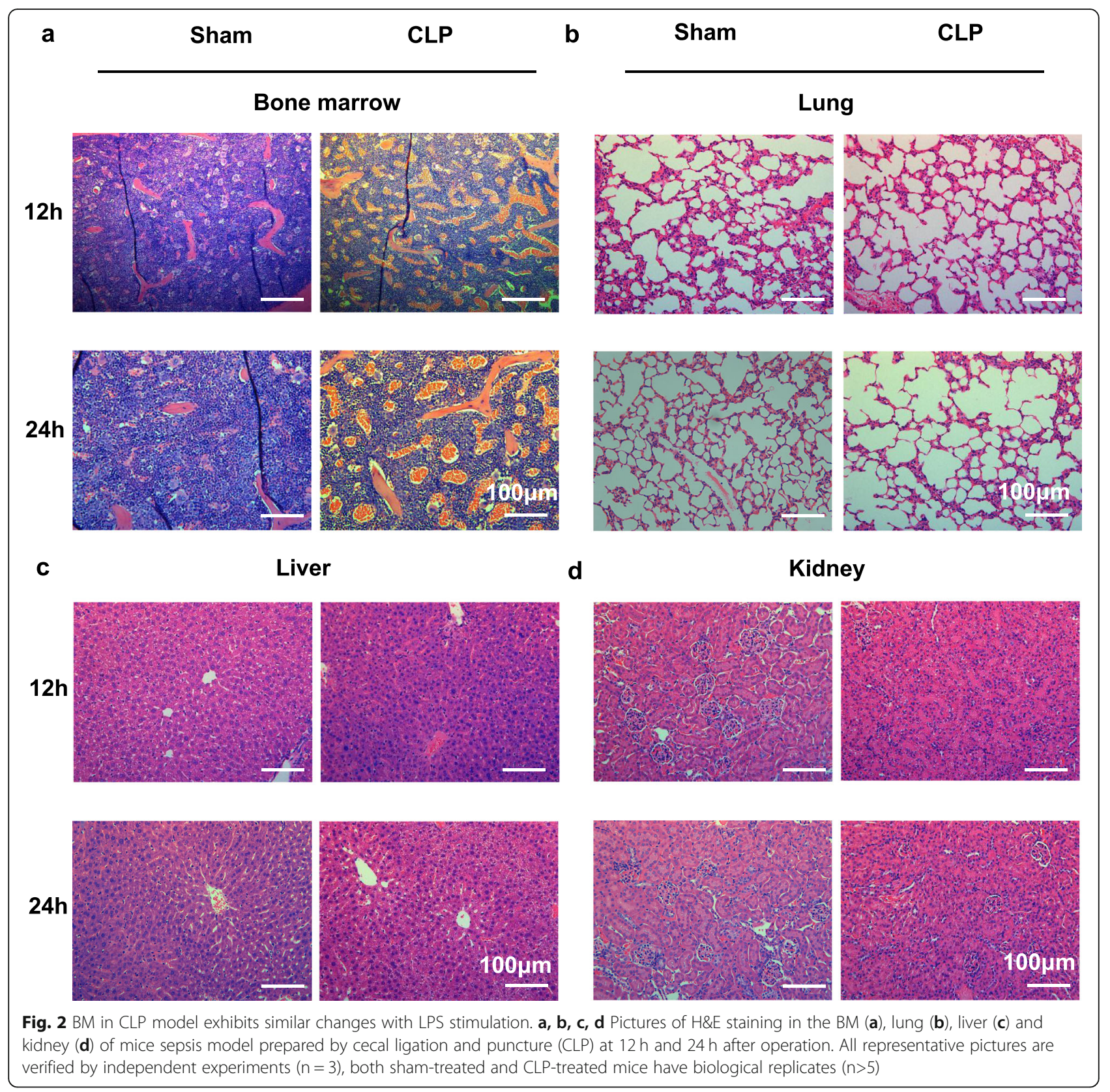

\section{Sepsis-mediated BM immune changes have a different immunophenotype than the periphery}

In our research, we focused on the changes of BM T lymphocytes subsets, especially regulatory $\mathrm{T}$ cell. After $24 \mathrm{~h}$, LPS treated mice had a higher level of $\mathrm{CD}^{+} \mathrm{CD}^{+} \mathrm{T}$ cells and $\mathrm{CD}^{+}{ }^{+} \mathrm{CD}^{+}{ }^{+} \mathrm{T}$ cells in $\mathrm{BM}$ compared with PBS treated mice, meanwhile the absolute numbers of these two cells also increased significantly in LPS treated mice (Fig. 5a, b). On the contrary, $\mathrm{CD}^{+} \mathrm{CD} 4^{+} \mathrm{T}$ cells in spleen of LPS treated mice show a significant increase while $\mathrm{CD}^{+} \mathrm{CD} 8^{+}$ $\mathrm{T}$ cells showed no obvious changes. Taken together peripheral lymphocytes subsets reveal a different pattern of immune response from BM (Fig. 5a, c).
We further analyzed the changes of Treg cells, an important inhibitory component of CD4 lymphocytes subsets in spleen and BM after LPS treatment. It is worth noting that BM Treg cells and spleen Treg cells also showed different response patterns after LPS stimulation. The percentage of $\mathrm{BM} \mathrm{FOXP3}^{+}$Treg cells decreased significantly in LPS treated mice compared with PBS treated mice (Fig. 5d, f). Inversely, $\mathrm{FOXP3}^{+}$Treg cells showed an increase in spleen of mice treated with LPS (Fig. 5e, g). Notably, unlike the transient changes of BM ECs, the LPS induced Treg cells changes in $\mathrm{BM}$ and spleen are more durable. These results collectively suggest that the BM immune niche has a different response pattern from the peripheral immune organ in sepsis. 
a
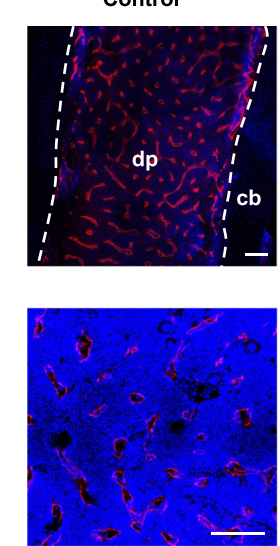

b

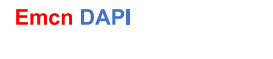

\section{LPS D1}
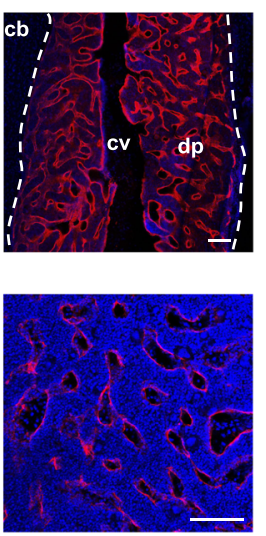

c
Sham
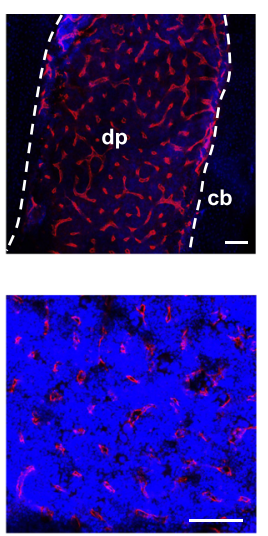

d

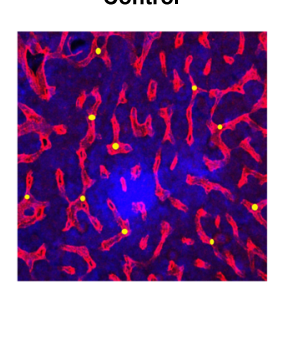

CLP D1
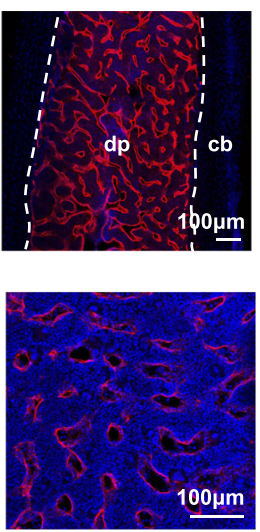

LPS D1
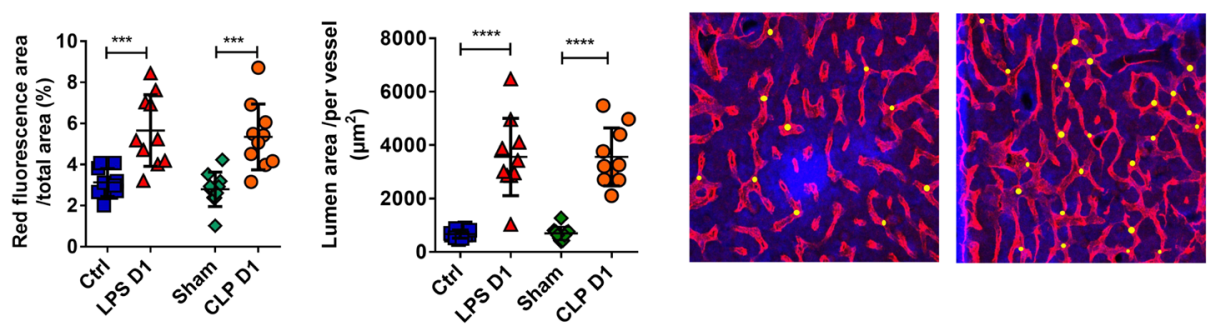

e

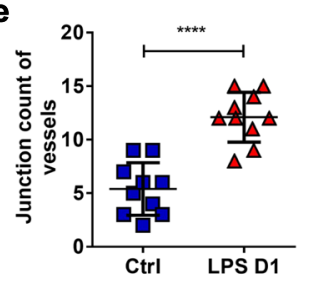

f

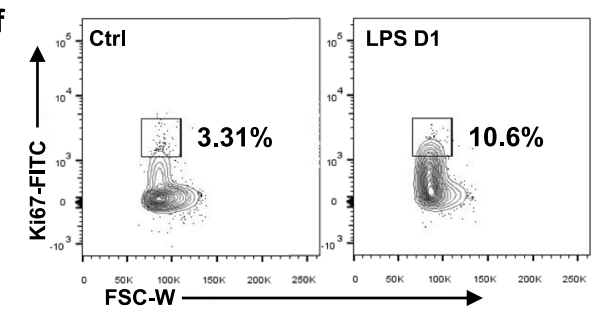

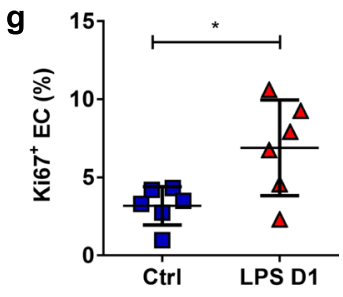
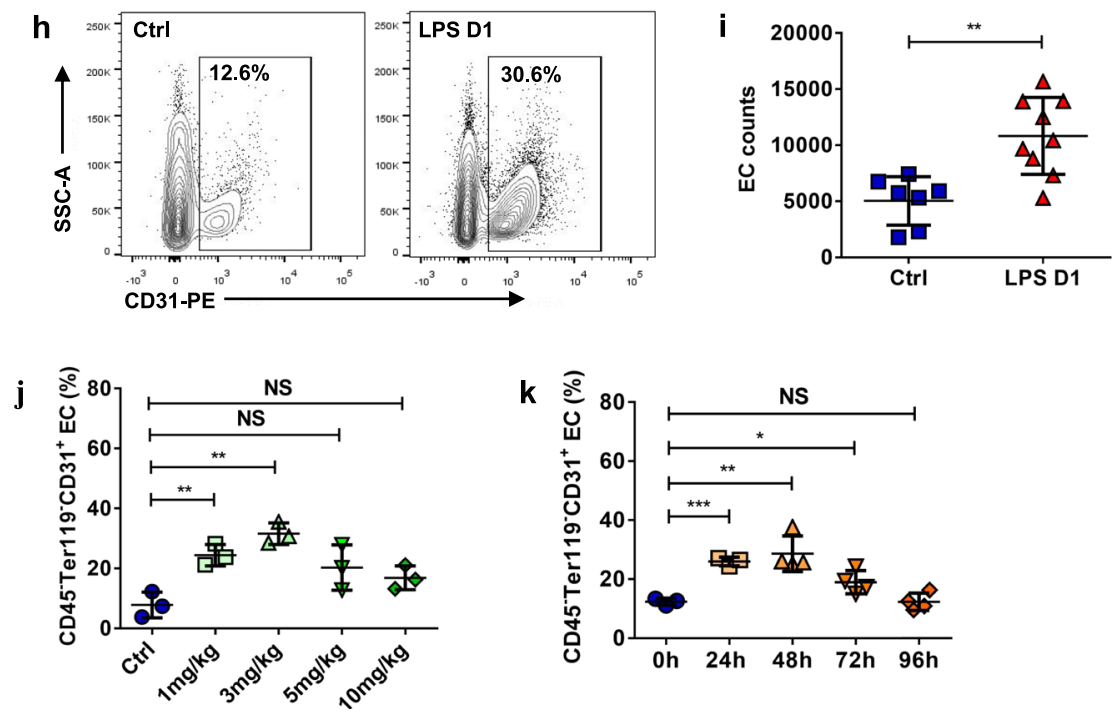

Fig. 3 (See legend on next page.) 


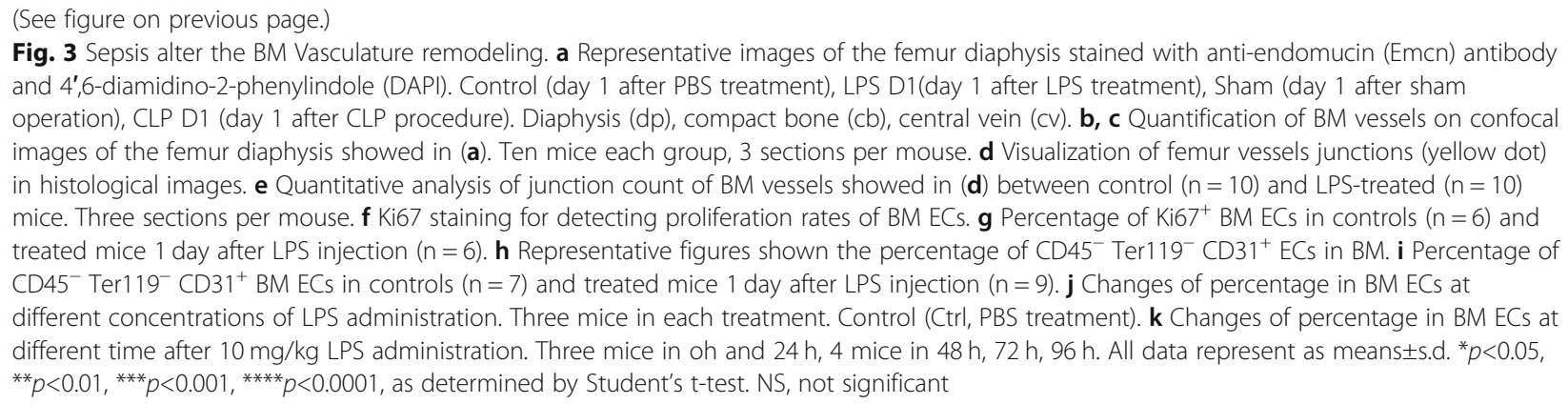

\section{Discussion}

Sepsis-mediated changes in BM niche components could have a profound effect on hematopoiesis, and may also be one of the pathogenesis of sepsis. In this study. We describe the significant changes of major components in $\mathrm{BM}$ niche during early stage of sepsis.

One of the challenges in research related to sepsis is the limitations and variability of existing models of sepsis in simulating human pathophysiologic conditions [12]. CLP is the preferred approach for modeling sepsis, reflecting progression of sepsis characterized with systemic inflammatory response and the compensatory anti-inflammatory response [13]. LPS is the main glycolipid present in the outer leaflet of the outer membrane of Gram-negative bacteria which can induce a strong inflammatory response. We found that organic pathological changes in mice were similar between the two models, and major pro-inflammatory factors upregulated significantly. In addition, lethal dose of LPS injection usually induces severe inflammatory response and aggravates organ damage, allowing better observation of phenotypes in early stage of sepsis. In this study, sepsis model was mainly performed by lethal does LPS intraperitoneal injection. Because it is worth noting that LPS-induced sepsis model fit the early stage of sepsis and the surviving mice gradually recovered from LPS challenge in the later period (several weeks).

BM ECs are the gateway against infection, so understanding the impact of inflammation on the BM vasculature is essential. Some evidence suggests that the BM vasculature undergoes significant remodeling under LPS stimulation [14]. We found for the first time that after LPS stimulation, BM ECs responded quickly, with a transient increase, and this change did not depend on the dose of LPS administered. The rapid and transient activation of BM ECs may be an emergency response to inflammatory signals from the hematopoietic system, and this effect may in turn promote the maintenance of $\mathrm{BM}$ homeostasis. In addition, this transient change in ECs may also have temporal and spatial consistency with certain physiological processes during inflammation. For example, neutrophils migrate from the $\mathrm{BM}$ to the periphery during inflammation [15]. Platelet activation and VEGF signaling are the basic mediators of EC activation during inflammation $[16,17]$. However, in our study, VEGF expression in ECs was not up-regulated with LPS stimulation. This may indicate that endothelial-derived VEGF is not a major factor in the remodeling of the vasculature during inflammation. This regulation may come from other cells in the BM and platelets.

MSCs are major components of the BM niche, which contain multiple and overlapping populations depending on different markers and genetic reporters [18]. NestinGFP as one of the genetic reporters is used to label and trace MSCs [11]. In our study, we used nestin-GFP transgenic mice to assess the effect of sepsis on MSCs in niche. Unlike hematopoietic stem cells, MSCs cannot be mobilized into the peripheral circulation which respond to inflammatory signals generated locally or systemically in situ [19]. MSC senses inflammation stress through pattern recognition receptor (PRR) modifying the cytokine and chemokine profile in BM stroma, which result in a series of adaptive effect including hematopoietic changes [20], mobilization of HSCs [21], monocyte release [9] and inhibition of neutrophil apoptosis [22]. The importance of MSCs as a component of the niche under inflammation condition on stroma remodeling and hematopoietic support is self-evident. However, little is known about the effects of inflammation on MSCs. We report no significant increase in frequency and absolute number with unchanged spatial localization of MSCs during sepsis. MSCs appear to be in a resting state of morphology, which is not consistent with their functional activation during inflammation. In addition, our study failed to address the changes in MSCs throughout sepsis.

Immunosuppression is one of the most important causes of mortality in sepsis [23], which is related to the inappropriate increase of Treg cells and the enhanced function [24, 25]. BM Tregs are an important niche component [26-29] and represent a unique T-cell lineage that the frequency of BM Tregs is significantly increased compared to the periphery [30, 31], and exhibits high inhibitory activity. TIGHT and CD44 and CXCR4 were highly expressed in BM Tregs [30]. BM 


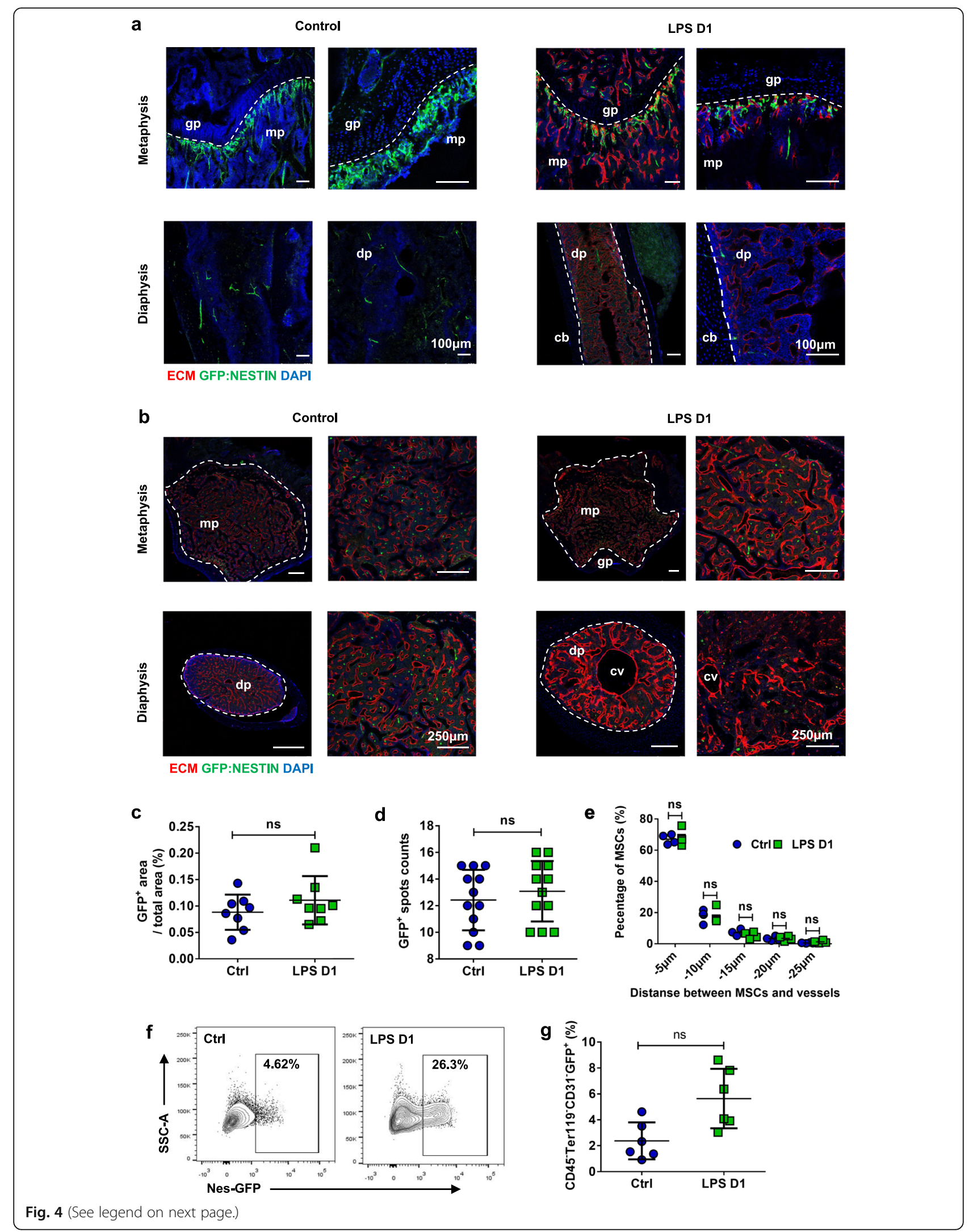


(See figure on previous page.)

Fig. 4 BM MSCs show no significant changes during sepsis. a, b Longitudinally (a) and transverse-shaved (b) confocal images of femur metaphysis and diaphysis stained with anti-Emcn antibody and DAPI in control (PBS treatment) and LPS-treated Nestin-GFP transgenic mice. Diaphysis (dp), metaphysis (mp), central vein (cv), growth plate (gp). c, d Quantification of BM GFP ${ }^{+}$MSCs on enlarged view showed in (b). Eight mice each group, 3 sections per mouse. e Quantification of distance between MSCs and BM vessels from femur transverse-shaved sections of control (PBS treatment) and LPS-treated Nestin-GFP mice 1 day after intervention. $\mathbf{f}$ Representative FACS figures showed the percentage of BM MSCs in control (PBS injection) and LPS-treated Nestin-GFP transgenic mice 1 day after intervention. $\mathbf{g}$ Percentage of CD45-Ter $119^{-} \mathrm{CD}^{-} 1^{-} \mathrm{GFP}^{+}$ MSCs in control $(n=6)$ and LPS treated $(n=6)$ mice 1 day after intervention. All data represent as means $\pm s$.d. NS, not significant

Treg has been considered to be involved in the pathophysiological processes of various diseases, such as GVHD and prostate cancer [31, 32]. Whether BM Tregs functions in sepsis remain unclear. We observed a significantly decreased frequency of BM Tregs and an increased proportion of $\mathrm{CD}_{4}^{+} \mathrm{CD}^{+}$effector T cells in $\mathrm{BM}$ during sepsis. Notably, the peripheral $\mathrm{T}$ cell subsets changed completely opposite to BM. Tregs are known to be regulated by various chemokine regulatory axes, MSC in BM produce high levels of CXCL12 [30, 31], activated Tregs can migrate through their enhanced CXCR4 expression [33] and eventually reside in the BM. The upregulated expression of G-CSF in sepsis will reduce the concentration of BM CXCL12, which may cause the unstable of CXCL12/CXCR4 axis [34]. The disruption of this pathway mobilizes Tregs into the periphery, which account for the reduction of BM Tregs and the differentiation and expansion of effector $\mathrm{CD} 4^{+}$and $\mathrm{CD} 8^{+} \mathrm{T}$ cells. It should be noted that sepsis-induced BM Treg changes may lead to the destruction of the HSC immune privilege status [35], resulting in a series of hematopoietic problems in the BM under the inflammatory state.

Our findings map the response of major BM niche components to sepsis, which reflects in the earlier and more prominent histological changers than other organs and urgent mobilization of non-hematopoietic cells, such as ECs and Tregs, in the BM under sepsis. We put forward a hypothesis that BM may be the initiating or accelerating organ during sepsis. Future studies will delve into the cellular changes of niche components and study the relationships among BM niche components and peripheral organic microenvironment under the inflammatory state, which may reveal the critical mechanism of septic onset and progress.

\section{Materials and methods}

\section{Animals}

Sepsis models were performed in several mouse strains: adult C57BL/6 mice (Shanghai SLAC Laboratory Animal Co., Ltd., Shanghai, China); Nestin-GFP and Sca1-GFP transgenic mice (The Jackson Laboratory, Bar Harbor, ME, USA). Sexmatched mice of both sexes between the ages of 8 and 14 weeks were used.

\section{Sepsis model}

Sepsis model was performed by LPS intraperitoneal injection [36] and cecal ligation puncture (CLP) according to the methods described in previous studies $[8,13]$. Briefly, for CLP model, cecum was ligated at half the distance between distal pole and the base of the cecum with $18 \mathrm{G}$ needle to induce abdominal infection, resulting in a mortality of $60 \%$ on the seventh day. The control group underwent exactly the same procedures except for the CLP. For LPS intraperitoneal injection model, mice were subjected to intraperitoneal injection of $10 \mathrm{mg} / \mathrm{kg}$ of $E$. coli serotype O111:B4 lipopolysaccharide (LPS) purchased from Sigma (L 4130). Mice in control group was injected with PBS which was used to dissolve LPS. The survival curves of both models were highly matched.

\section{Hematoxylin-eosin staining}

For histology, dissected femurs were fixed in 10\% neutral buffered formalin overnight, followed by decalcification in $10 \%$ EDTA for 2 weeks. Femurs were embedded in and then sectioned at $6 \mu \mathrm{m}$ thickness. Hematoxylin and eosin (H\&E) staining was performed according to a standard procedure [37].

\section{Immunostaining}

Immunostaining was performed according to a published procedure [38]. Briefly, dissected femurs were fixed with $4 \%$ paraformaldehyde (PFA) at $4{ }^{\circ} \mathrm{C}$ overnight and decalcified in $0.5 \mathrm{M}$ EDTA for $24 \mathrm{~h}$ with constantly shaking. The femurs were dehydrated with $20 \%$ sucrose at $4^{\circ} \mathrm{C}$ for $24 \mathrm{~h}$ then embedded and stored at $-80^{\circ} \mathrm{C}$ overnight before section. Femurs were sectioned horizontally and longitudinally at $40 \mu \mathrm{m}$ thickness using Leica cryostat (Leica, CM1950). For immunostaining, femur sections were stained with Endomucin (anti-rat, sc-65,495, Santa Cruz Biotech.). The primary antibodies were bound by secondary antibodies (AF555 goat anti-rat, 2,018,295, Life technologies). Nuclei were stained with DAPI $(62,248$, Life Technologies). Images were acquired with a confocal microscope (Leica SP8) and analyzed with ImageJ and Angio Tool software. 

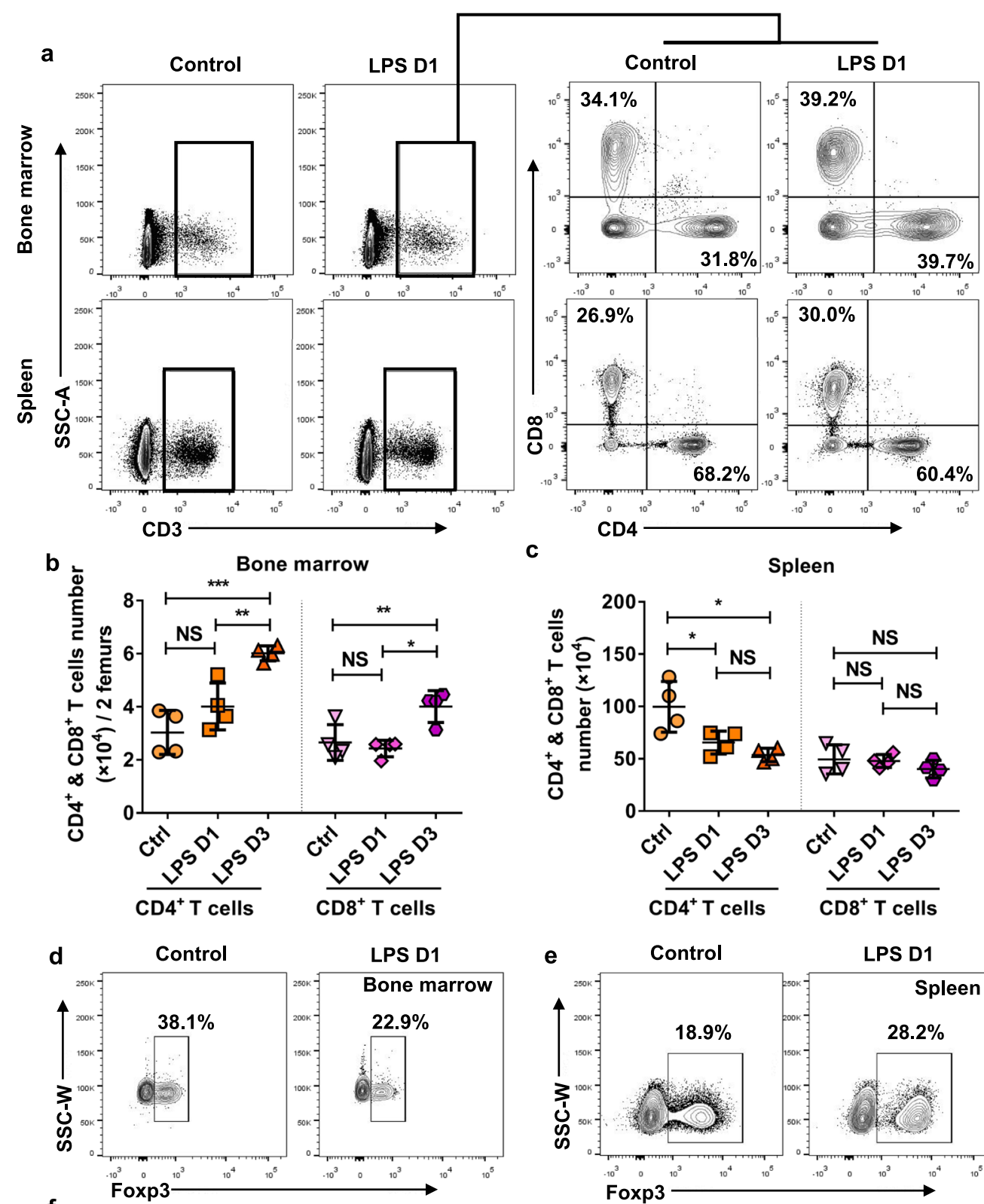

f
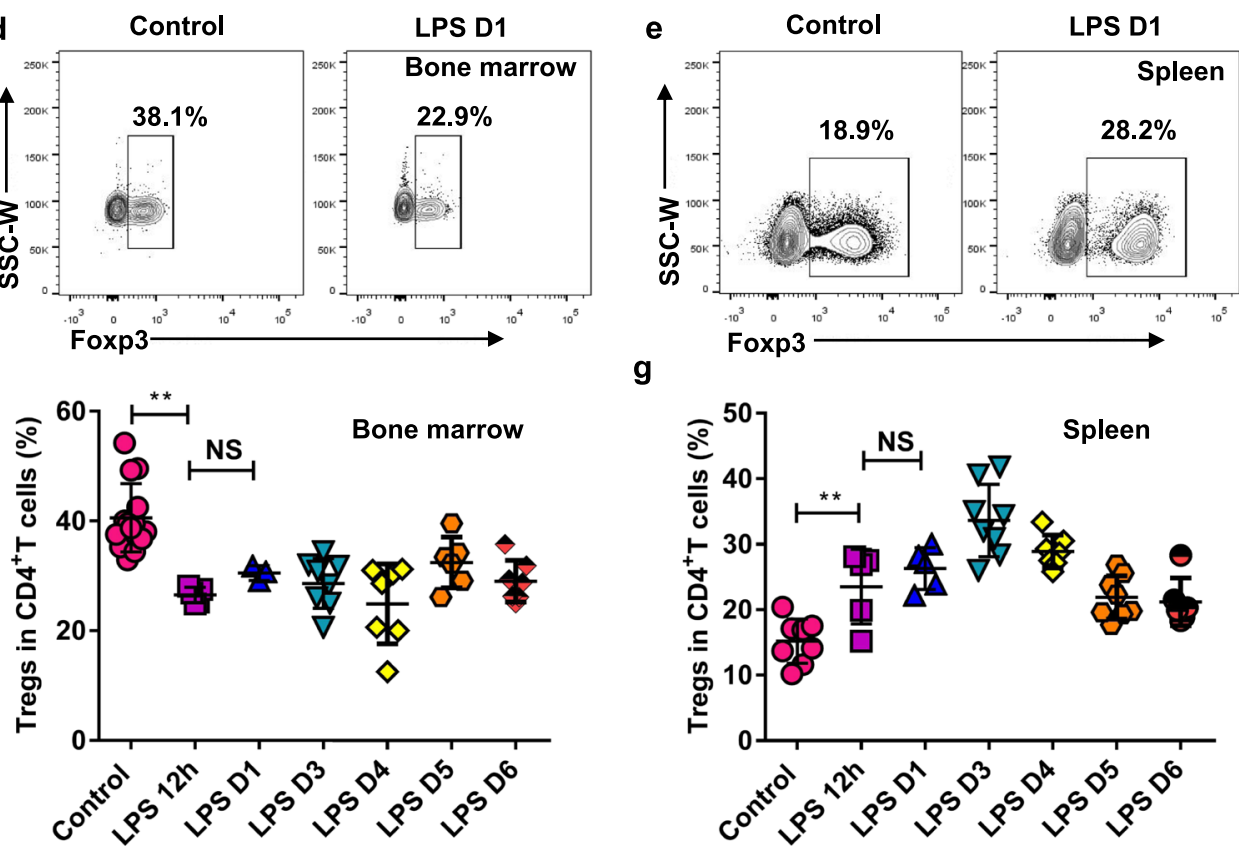

Fig. 5 (See legend on next page.) 
(See figure on previous page.)

Fig. 5 BM immune cells exhibit different characteristics from the periphery and show complexity changes during sepsis. a Representative figures shown the percentage of $\mathrm{CD}^{+} \mathrm{CD}^{-}$and $\mathrm{CD} 4^{-} \mathrm{CD} 8^{+} \mathrm{T}$ cells in the BM and spleen of control (PBS-treated, $\left.n=4\right)$ and LPS-treated mice $(n=4)$ at day 1. b, c Absolute cell counts of $C D 4^{+} C D 8^{-}$and $C D 4^{-} C D 8^{+} T$ cells in $\mathrm{BM}(\mathbf{b})$ and spleen (c) as shown in (a) at day1 and day 3. $\mathbf{d}, \mathbf{e}$ Representative figures shown the percentage of $\mathrm{CD}^{+} \mathrm{CD}^{+} \mathrm{FOXP}^{+}$regular T cells (Tregs) in the BM (d) and spleen (e) of control (PBS-treated) and LPS-treated mice at day 1. f, $\mathbf{g}$ Dynamic changes of the percentage of Treg cells within a week after LPS treatment. All data represent as means \pm s.d. ${ }^{*} p<0.05,{ }^{* *} p<0.01,{ }^{* * *} p<0.001,{ }^{* * *} p<0.0001$, as determined by Student's t-test. NS, not significant

\section{Flow cytometry}

For flow cytometry of ECs and MSCs, bones (Tibia, femur, sacrum) were crushed and digested with $1 \mathrm{mg} /$ $\mathrm{mL}$ collagenase IV and $2 \mathrm{mg} / \mathrm{mL}$ dispase in phosphate buffer saline (PBS) containing $1 \%$ BSA at $37^{\circ} \mathrm{C}$ for $1 \mathrm{~h}$. For immune cells, bones were flushed with pre-chilled PBS. For Single-cell suspensions of peripheral immune organs, lymph nodes and spleens were crushed gently on filter. Collected cells were incubated with Fc blocker anti-CD16/32 antibody (93, 14-0161-82) for $15 \mathrm{~min}$ on ice, followed by staining with fluorochrome-conjugated antibody on ice for $45 \mathrm{~min}$. The antibody used in this study included FITC/APC anti-CD45 (30-F11, 11-045185/17-0451-82); FITC/APC anti-Ter119 (Ter-119, 115921-82/17-5921-82); APC/PE anti-CD31 (390, 120311-82/17-0311-82) (all from ebioscience); DAPI (4',6diamino-2-phenylindole) (62,248, Life Technologies) was used to exclude dead cells. BD FACS Canto II flow cytometer equipped with the FACS Diva 6.1 software (BD Biosciences) were used to collect samples information. Data were analyzed with FlowJo version 10.

\section{Statistical analysis}

All data are measurement data and represented as mean \pm s.e.m. Normality analysis (Kolmogorov-Smirnov tests) and homogeneity test of variance (Levene tests) were done between different samples, Comparisons between two samples were done using the unpaired Student's t tests. One-way ANOVA analyses followed by Student-Newman-Keuls multiple comparison tests were used for multiple group comparisons. Wilcoxon tests and Kruskal-Wallis tests were used for samples with inconsistent distributions and variances. Statistical analyses were performed with GraphPad Prism 6 and Statistical Product and Service Solutions (SPSS) version 20 software. ${ }^{*} p<0.05,{ }^{* * *} p<0.01$, ${ }^{* * * *} p<0.001$.

\section{Abbreviations}

FACS: Fluorescence activated cell sorting; BM: Bone marrow; LPS: Lipopolysaccharide; CLP: Cecal ligation and puncture; ECs: Endothelial cells; GFP: Green fluorescent protein; MSCs: Mesenchymal stem cells; Tregs: Regulatory T cells; FOXP3: Forkhead box protein 3

\section{Acknowledgements}

We thank Wei-Wei Zheng, Da-Bing Tang (Shanghai Children's Medical Center, Shanghai Jiao Tong University School of Medicine) for excellent technical support.

\section{Consent to participate}

This research does not involve human studies, "consent to participate" is not applicable.

\section{Code availability}

Not applicable.

\section{Authors' contributions}

Botao Ning and Caiwen Duan designed and supervised the study. Fan Yin and Han Qian performed experiments, interpreted and analyzed data. Fan Yin wrote the paper. The authors read and approved the final manuscript.

\section{Funding}

The work is supported by the Shanghai Natural Science Foundation of China (No. 19ZR1432900).

\section{Availability of data and materials}

All data generated or analyzed during the current study are available from the corresponding author on reasonable request.

\section{Ethics approval}

All studies on animals were performed after approval by the Ethics Committee of Shanghai Children's Medical Center, in compliance with Guidelines for the Use and Care of Small Laboratory Animals.

\section{Consent for publication}

Written informed consent for publication was obtained from all participants.

\section{Competing interests}

The authors declare that they have no competing interests.

Received: 14 August 2020 Accepted: 28 September 2020

Published online: 20 October 2020

\section{References}

1. Hotchkiss RS, Moldawer LL, Opal SM, Reinhart K, Turnbull IR, Vincent JL. Sepsis and septic shock. Nat Rev Dis Primers. 2016;2:16045. https://doi.org/ 10.1038/nrdp.2016.45.

2. Fleischmann C, Scherag A, Adhikari NK, Hartog CS, Tsaganos T, Schlattmann $P$, et al. Assessment of global incidence and mortality of hospital-treated sepsis. Current estimates and limitations. Am J Respir Crit Care Med. 2016; 193(3):259-72. https://doi.org/10.1164/rccm.201504-07810C.

3. Lelubre C, Vincent J-L. Mechanisms and treatment of organ failure in sepsis. Nat Rev Nephrol. 2018;14(7):417-27. https://doi.org/10.1038/s41581-0180005-7.

4. Bellomo R, Kellum JA, Ronco C, Wald R, Martensson J, Maiden M, et al. Acute kidney injury in sepsis. Intensive Care Med. 2017;43(6):816-28. https:// doi.org/10.1007/s00134-017-4755-7.

5. Haak BW. Wiersinga WJ. The role of the gut microbiota in sepsis. Lancet Gastroenterol Hepatol. 2017;2(2):135-43. https://doi.org/10.1016/s24681253(16)30119-4.

6. Capan M, Hoover S, Ivy JS, Miller KE, Arnold R. Not all organ dysfunctions are created equal - prevalence and mortality in sepsis. J Crit Care. 2018;48: 257-62. https://doi.org/10.1016/j.jcrc.2018.08.021

7. Venet F, Demaret J, Gossez M, Monneret G. Myeloid cells in sepsis-acquired immunodeficiency. Ann N Y Acad Sci. 2020. https://doi.org/10.1111/nyas. 14333.

8. Rodriguez S, Chora A, Goumnerov B, Mumaw C, Goebel WS, Fernandez L, et al. Dysfunctional expansion of hematopoietic stem cells and block of myeloid differentiation in lethal sepsis. Blood. 2009;114(19):4064-76. https:// doi.org/10.1182/blood-2009-04-214916. 
9. Shi C, Jia T, Mendez-Ferrer S, Hohl TM, Serbina NV, Lipuma L, et al. Bone marrow mesenchymal stem and progenitor cells induce monocyte emigration in response to circulating toll-like receptor ligands. Immunity. 2011;34(4):590-601. https://doi.org/10.1016/j.immuni.2011.02.016.

10. Hassoun PM, Mouthon L, Barberà JA, Eddahibi S, Flores SC, Grimminger F, et al. Inflammation, growth factors, and pulmonary vascular remodeling. J Am Coll Cardiol. 2009;54(1 Suppl):S10-9. https://doi.org/10.1016/j.jacc.2009.04.006.

11. Mendez-Ferrer S, Michurina TV, Ferraro F, Mazloom AR, Macarthur BD, Lira SA, et al. Mesenchymal and haematopoietic stem cells form a unique bone marrow niche. Nature. 2010;466(7308):829-34. https://doi.org/10.1038/ nature09262.

12. Rittirsch D, Hoesel LM, Ward PA. The disconnect between animal models of sepsis and human sepsis. J Leukoc Biol. 2007;81(1):137-43. https://doi.org/ 10.1189/jlb.0806542.

13. Rittirsch D, Huber-Lang MS, Flierl MA, Ward PA. Immunodesign of experimental sepsis by cecal ligation and puncture. Nat Protoc. 2009;4(1): 31-6. https://doi.org/10.1038/nprot.2008.214

14. Vandoorne K, Rohde D, Kim HY, Courties G, Wojtkiewicz G, Honold L, et al. Imaging the vascular bone marrow niche during inflammatory stress. Circ Res. 2018;123(4):415-27. https://doi.org/10.1161/circresaha.118.313302.

15. Borregaard N. Neutrophils, from marrow to microbes. Immunity. 2010;33(5): 657-70. https://doi.org/10.1016/j.immuni.2010.11.011.

16. Lee $\mathrm{S}$, Chen $\Pi$, Barber $\mathrm{CL}$, Jordan MC, Murdock J, Desai S, et al. Autocrine VEGF signaling is required for vascular homeostasis. Cell. 2007;130(4):691703. https://doi.org/10.1016/j.cell.2007.06.054.

17. Stokes KY, Granger DN. Platelets: a critical link between inflammation and microvascular dysfunction. J Physiol. 2012;590(5):1023-34. https://doi.org/10. 1113/jphysiol.2011.225417.

18. Batsivari A, Haltalli MLR, Passaro D, Pospori C, Celso CL, Bonnet D. Dynamic responses of the haematopoietic stem cell niche to diverse stresses. Nat Cell Biol. 2020;22(1):7-17. https://doi.org/10.1038/s41556-019-0444-9.

19. Tyndall A, Pistoia V. Mesenchymal stem cells combat sepsis. Nat Med. 2009; 15(1):18-20. https://doi.org/10.1038/nm0109-18.

20. Chou DB, Sworder B, Bouladoux N, Roy CN, Uchida AM, Grigg M, et al. Stromal-derived IL-6 alters the balance of myeloerythroid progenitors during toxoplasma gondii infection. J Leukoc Biol. 2012;92(1):123-31. https://doi.org/10.1189/jlb.1011527.

21. Petit I, Szyper-Kravitz M, Nagler A, Lahav M, Peled A, Habler L, et al. G-CSF induces stem cell mobilization by decreasing bone marrow SDF-1 and up-regulating CXCR4. Nat Immunol. 2002;3(7):687-94. https://doi.org/10.1038/ni813.

22. Raffaghello L, Bianchi G, Bertolotto M, Montecucco F, Busca A, Dallegri F, et al. Human mesenchymal stem cells inhibit neutrophil apoptosis: a model for neutrophil preservation in the bone marrow niche. Stem Cells. 2008; 26(1):151-62. https://doi.org/10.1634/stemcells.2007-0416.

23. Boomer JS, To K, Chang KC, Takasu O, Osborne DF, Walton AH, et al. Immunosuppression in patients who die of sepsis and multiple organ failure. Jama. 2011;306(23):2594-605. https://doi.org/10.1001/jama.2011.1829.

24. Delano MJ, Ward PA. The immune system's role in sepsis progression, resolution, and long-term outcome. Immunol Rev. 2016;274(1):330-53. https://doi.org/10.1111/imr.12499.

25. Kessel A, Bamberger $\mathrm{E}$, Masalha M, Toubi E. The role of T regulatory cells in human sepsis. J Autoimmun. 2009;32(3-4):211-5. https://doi.org/10.1016/j. jaut.2009.02.014

26. Wei S, Kryczek I, Zou W. Regulatory T-cell compartmentalization and trafficking. Blood. 108(2):426-31. https://doi.org/10.1182/blood-2006-01-0177.

27. Hirata Y, Furuhashi K, Ishii H, Li HW, Pinho S, Ding L, et al. CD150(high) Treg cells may attenuate graft versus host disease and intestinal cell apoptosis after hematopoietic stem cell transplantation. Am J Transl Res. 2019;11(3): 1299-310. https://doi.org/10.1016/j.stem.2018.01.017.

28. Hirata Y, Furuhashi K, Ishii H, Li HW, Pinho S, Ding L, et al. CD150(high) bone marrow Tregs maintain hematopoietic stem cell quiescence and immune privilege via adenosine. Cell Stem Cell. 2018;22(3):445-453.e445. https://doi.org/10.1016/j.stem.2018.01.017.

29. Pierini A, Nishikii H, Baker J, Kimura T, Kwon H-S, Pan Y, et al. Foxp3 regulatory $T$ cells maintain the bone marrow microenvironment for $B$ cell lymphopoiesis. Nat Commun. 2017:8:15068. https://doi.org/10.1038/ ncomms15068.

30. Zou L, Barnett B, Safah H, Larussa VF, Evdemonhogan M, Mottram P, et al. Bone marrow is a reservoir for CD4+CD25+ regulatory $T$ cells that traffic through CXCL12/CXCR4 signals. Cancer Res. 2004;64(22):8451-5. https://doi. org/10.1158/0008-5472.CAN-04-1987.
31. Zhao E, Wang L, Dai J, Kryczek I, Wei S, Vatan L, et al. Regulatory T cells in the bone marrow microenvironment in patients with prostate cancer. Oncoimmunology. 2012;1(2):152-61. https://doi.org/10.4161/onci.1.2.18480.

32. Rezvani K, Mielke S, Ahmadzadeh M, Kilical Y, Savani BN, Zeilah J, et al. High donor FOXP3-positive regulatory T-cell (Treg) content is associated with a low risk of GVHD following HLA-matched allogeneic SCT. Blood. 2006; 108(4):1291-7. https://doi.org/10.1182/blood-2006-02-003996.

33. Wei S, Kryczek I, Edwards RP, Zou L, Szeliga W, Banerjee M, et al. Interleukin2 administration alters the CD4+FOXP3+ T-cell pool and tumor trafficking in patients with ovarian carcinoma. Cancer Res. 2007;67(15):7487-94. https:// doi.org/10.1158/0008-5472.CAN-07-0565.

34. Day RB, Bhattacharya D, Nagasawa T, Link DC. Granulocyte colonystimulating factor reprograms bone marrow stromal cells to actively suppress B lymphopoiesis in mice. Blood. 2015;125(20):3114-7. https://doi. org/10.1182/blood-2015-02-629444.

35. Fujisaki J, Wu J, Carlson AL, Silberstein L, Putheti P, Larocca R, et al. In vivo imaging of Treg cells providing immune privilege to the haematopoietic stem-cell niche. Nature. 2011;474(7350):216-9. https://doi.org/10.1038/ nature10160.

36. Opal SM. Endotoxins and other sepsis triggers. Contrib Nephrol. 2010;167: 14-24. https://doi.org/10.1159/000315915.

37. Fischer AH, Jacobson KA, Rose J, Zeller R. Hematoxylin and eosin staining of tissue and cell sections. CSH Protoc. 2008;2008:pdb.prot4986. https://doi. org/10.1101/pdb.prot4986.

38. Kusumbe AP, Ramasamy SK, Starsichova A, Adams RH. Sample preparation for high-resolution 3D confocal imaging of mouse skeletal tissue. Nat Protoc. 2015;10(12):1904-14. https://doi.org/10.1038/nprot.2015.125.

\section{Publisher's Note}

Springer Nature remains neutral with regard to jurisdictional claims in published maps and institutional affiliations. 